\title{
Cutaneous pigmented invasive squamous cell carcinoma: a case report with dermatoscopy and histology
}

\author{
Cliff Rosendahl, $\mathrm{MBBS}^{1}$, Alan Cameron, $\mathrm{MBBS}^{1}$, Agata Bulinska, M.D. ${ }^{1}$, David Weedon, M.D. ${ }^{2}$
}

${ }^{1}$ School of Medicine, The University of Queensland, Brisbane, Australia

${ }^{2}$ Sullivan and Nicolaides Pathology, Brisbane, Australia

Key words: pigmented, squamous cell carcinoma, invasive, dermatoscopy, histology

Citation: Rosendahl C, Cameron A, Bulinska A, Weedon D. Cutaneous pigmented invasive squamous cell carcinoma: a case report with dermatoscopy and histology. Dermatol Pract Concept 2011;1(1):14. http://dx.doi.org/10.5826/dpc.0101a14.

Editor: Alon Scope, M.D.

Received: January 27, 2011; Accepted: June 21, 2011; Published: October 31, 2011

Copyright: @2011 Rosendahl et al. This is an open-access article distributed under the terms of the Creative Commons Attribution License, which permits unrestricted use, distribution, and reproduction in any medium, provided the original author and source are credited.

Funding: None.

Competing interests: The authors have no conflicts of interest to disclose.

All authors have contributed significantly to this publication.

Corresponding author: Cliff Rosendahl, MBBS, PO Box 734, Capalaba, Queensland, Australia, 4157. Email: cliffrosendahl@bigpond.com.

\section{Introduction}

Cutaneous invasive pigmented squamous cell carcinoma (SCC) is rare. There have previously been only a few case reports or small case series in the English literature [1-13].

In the largest published study there were five cases of pigmented invasive SCC in 46,791 archived cases of invasive SCC (approximately $0.01 \%$ ) [2]. This compares to pigmented SCC in-situ (pigmented Bowen's disease) in which the largest study found 52 of 951 (5.5\%) consecutive cases of SCC in-situ to be pigmented [14].

The differential diagnosis of pigmented invasive SCC includes benign and malignant melanocytic neoplasms (melanoma and nevus) as well as pigmented types of benign and malignant epithelial neoplasms such as basal cell carcinoma (BCC), actinic keratosis (AK), SCC in-situ, seborrheic keratosis and melanoacanthoma [1, 12].

Our search has revealed five cases of pigmented invasive SCC with a description of dermatoscopy features [1, 9, 10, $12,13]$ and in none of those cases was the diagnosis predicted.

\section{Case report}

An 80 year-old man with Fitzpatrick type 2 skin, presented for a routine skin check. A new raised pigmented skin lesion $6 \mathrm{~mm}$ in diameter was discovered on his right cheek (Figure 1). It had not been observed at his previous examination six months earlier. The patient migrated to Australia at the age of 21 . He had a long history of both occupational (bulldozer driver) and recreational (weekend fisherman) sun exposure.

The past medical history of this patient included the treatment of multiple histopathologically confirmed skin malignancies including SCC, SCC in-situ, BCC, AK and one in-situ melanoma. None of the treated non-melanoma skin cancers had been pigmented. The majority of these skin lesions were on the head, neck and upper limbs with a minority on the back and lower limbs.

Close macroscopic examination of the lesion on his right cheek (Figure 1) revealed that there was prominent surface scale overlying the posterior portion. A bluish discoloration was observed within the lesion; the clinician considered 


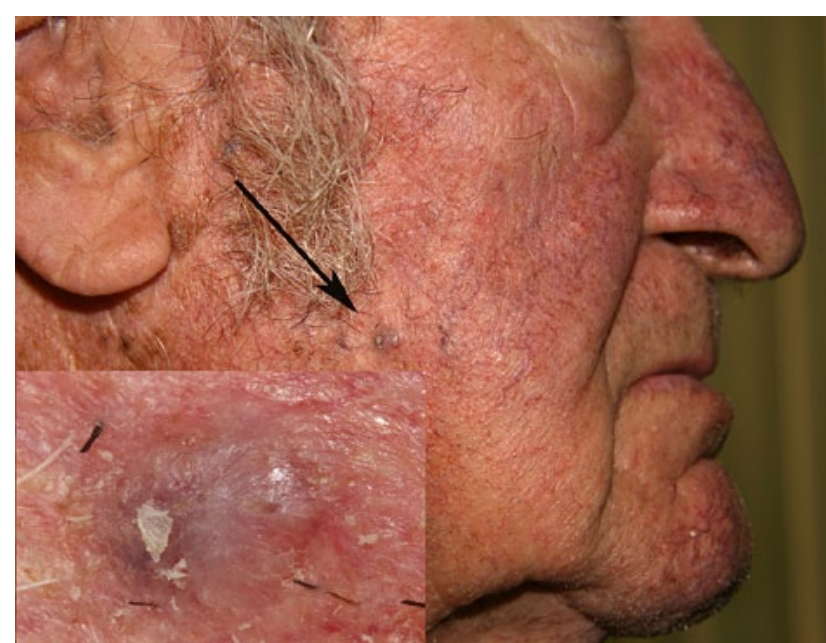

Figure 1. Clinical and macroscopic image of a raised pigmented lesion on the right cheek.

the source of this discoloration as the presence of melanin pigmentation. A smaller amount of scale was also present on peri-lesional skin. The lesion was raised and palpation revealed that it was nodular and slightly tender.

The surface of the lesion was tape-stripped to remove surface scale and examined with a non-polarizing dermatoscope. A photograph was taken (Figure 2) with a nonpolarizing Dermlite Fluid dermatoscope (3-Gen, San Juan Capistrano, CA) coupled to a Canon 50D single lens reflex camera(Canon, Tokyo, Japan).

Dermatoscopic features are described according to revised pattern analysis $[15,16]$.

Dermatoscopically (Figure 2) the peri-lesional skin was white in between the red vascular plexus. The overall pattern of the lesion was structureless. The anterior (Figure 2, right side) part of the lesion was blue superiorly with blue and white inferiorly and in these parts linear, serpentine, and coiled vessels were seen. Posteriorly (Figure 2, left side), denser blue pigment partially obscured the vessels. As an additional clue two white circles were present dermatoscopically (arrows, Figure 2). The peripheral borders of the lesion blended into peri-lesional skin without sharp demarcation. The lesion was excised in toto. It was signed out by the reporting pathologist as a "focally pigmented, moderately well differentiated acantholytic squamous cell carcinoma extending to mid-dermis." (Figures 3-5).

\section{Discussion}

A search of the literature reveals five previous reports with the dermatoscopic features of pigmented invasive SCC $[1,9$, $10,12,13]$.

In 1997 Kossard et al reported a case of a 79-year-old man with a $5 \mathrm{~mm}$ pigmented invasive SCC of the ear [1].

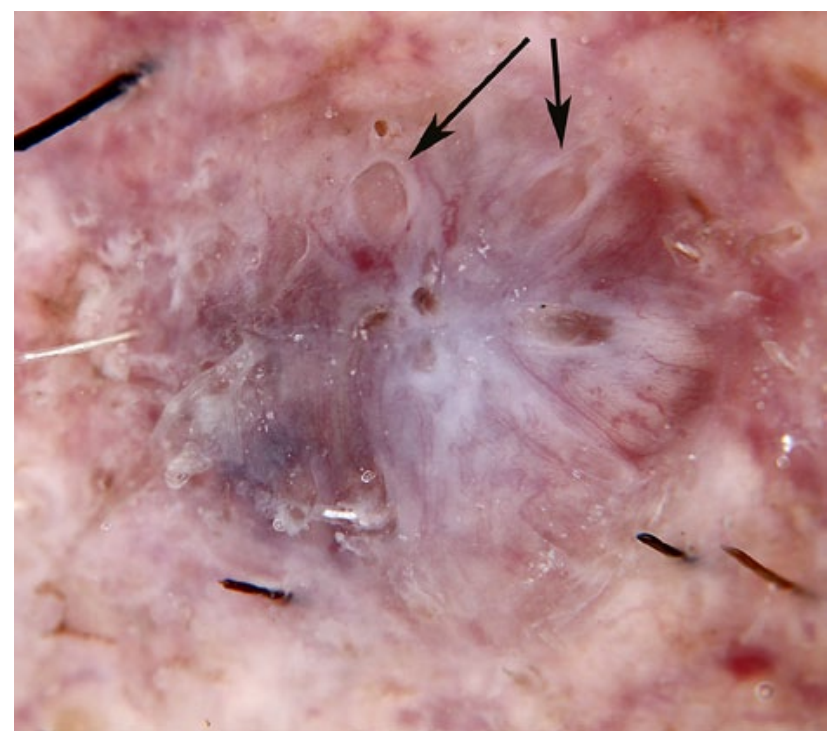

Figure 2. Dermatoscopic view of the lesion shown in Figure 1. Arrows point to white circles.

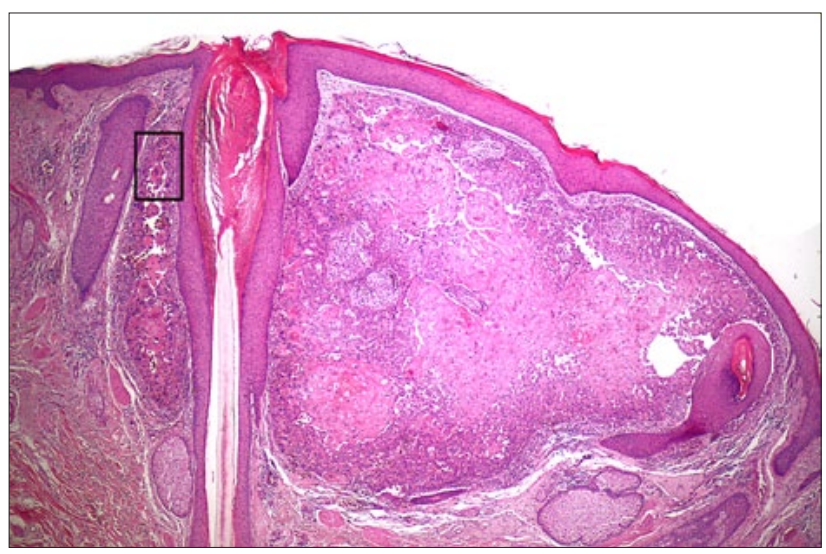

Figure 3. Histologic view (low power) of the lesion shown in Figures 1 and 2 shows invasive squamous cell carcinoma with aggregations of neoplastic keratinocytes, signs of abnormal cornification (eosinophilic cytoplasm and numerous dyskeratotic cells) and prominent acantholysis.

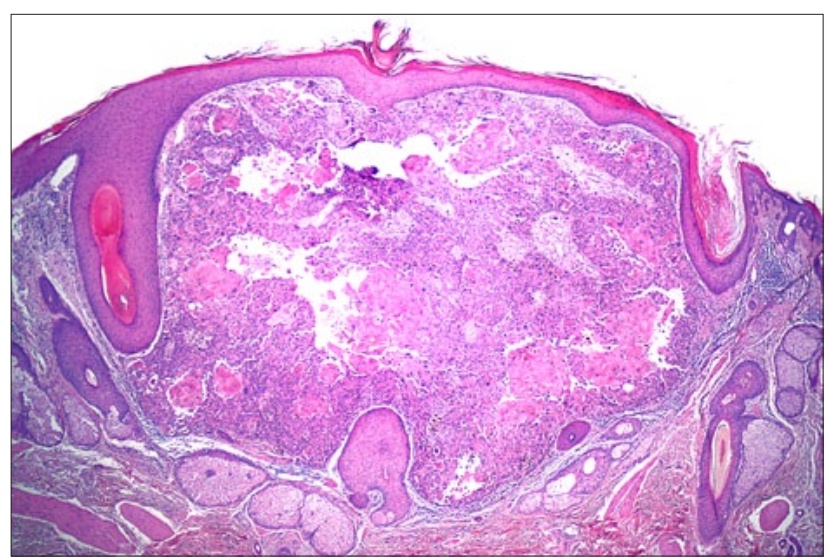

Figure 4. Additional low power histologic view. 


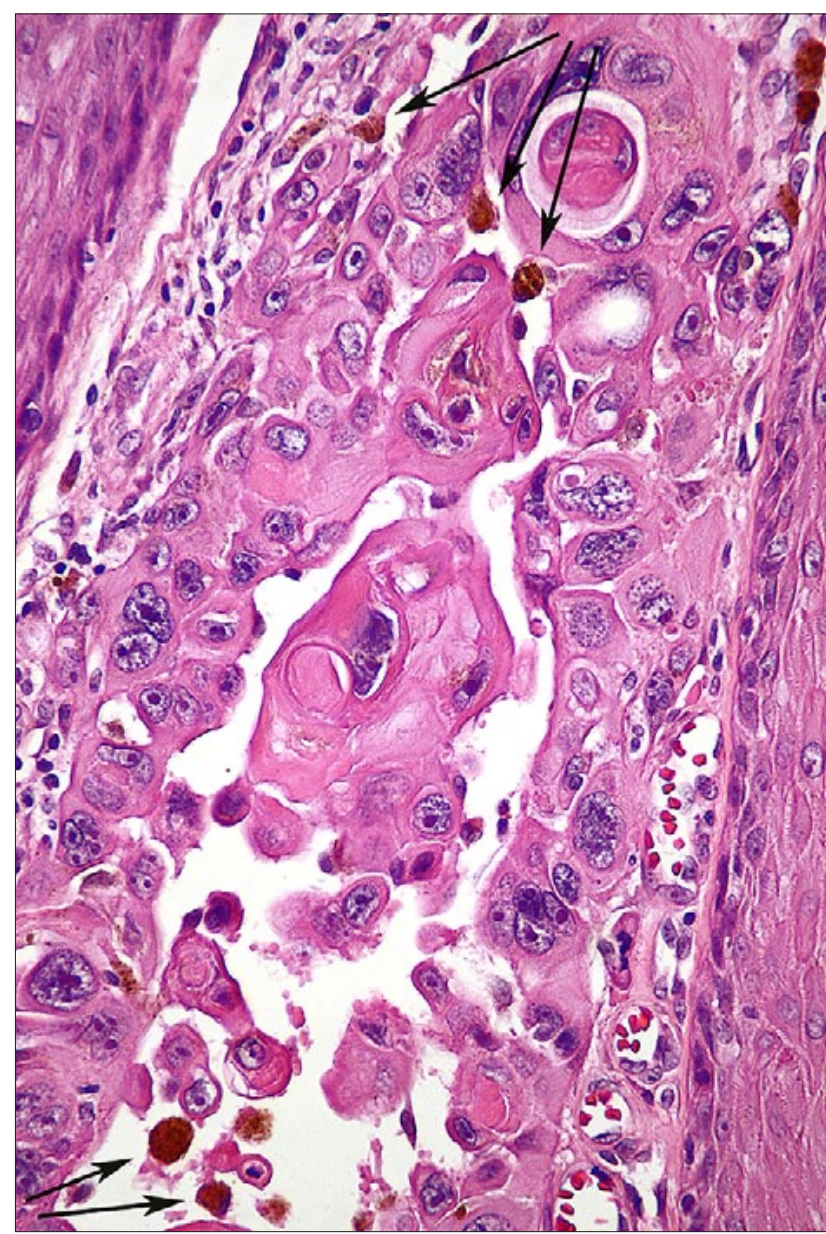

Figure 5. High power view of boxed area in Figure 3 showing abnormal keratinocytes with large nuclei and prominent nucleoli, dyskeratotic cells, melanin (arrows) and acantholysis.

They described the epiluminescent microscopy findings of "... a diffuse grey-blue colour, but the nodule lacked a peripheral pigment network and had no evident milia-like cysts." They considered a differential diagnosis of nodular melanoma, pigmented BCC, irritated seborrheic keratosis and thrombosed hemangioma.

In 2003 Ohnishi et al reported a case of a 91-year-old man with a deeply pigmented ulcerated SCC on the cheek [9]. Inspection of the dermatoscopic images presented revealed an asymmetric structureless lesion with the added clue to malignancy of the color blue [17]. There was no recorded prediction of the diagnosis in this case.

In 2004 Zalaudek et al reported a case on the chest of an 80-year-old white male [10]. Inspection of the dermatoscopic image presented showed a structureless blue lesion with white and grey areas corresponding to surface scale. The authors advised about the presence of ulceration but this is not evident in the dermatoscopic image. They considered the differential diagnosis of melanoma and pigmented BCC but not pigmented SCC.

In 2009 di Giorgi et al reported a case on the upper lip of a 45 -year-old woman [12]. Dermatoscopically there were no blue structures but there was a central red and brown structureless area with radial lines at the periphery in a circumferential distribution. In this case a dermatologist had previously made a diagnosis of seborrheic keratosis. After dermatoscopic assessment the authors made a provisional diagnosis of a "suspect melanocytic lesion."

Also in 2009, Yoshida et al reported a $2.2 \times 1.5 \mathrm{~cm}$ pigmented SCC on the nose of a 101-year-old Japanese woman [13]. They published dermatoscopic images which revealed a centrally ulcerated lesion with serpentine vessels and bordering the ulceration, a structureless pigmented area displaying predominantly blue color with some brown. Blue radial lines project focally at the periphery.

In the case we present here malignancy was predicted on the basis of the "chaos and clues" algorithm [17]: there was "chaos" (asymmetry) and the clues of "eccentric structureless zone (blue)" and "polymorphic vessels." Of the three pigmented malignancies (pigmented melanoma, pigmented BCC, and pigmented SCC), pigmented SCC should be considered because of the presence of surface scale (seen macroscopically) and white circles (seen dermatoscopically). The blue color was not consistent with pigmented SCC in-situ; the color blue indicated the presence of melanin in the deep dermis [18]. Therefore, invasive pigmented SCC was considered, and this was confirmed histopathologically.

\section{Conclusion}

Pigmented cutaneous invasive SCC is uncommon with few reports in the literature. Four out of five previously reported cases with dermatoscopy had a blue structureless zone. The case we report, like previous cases, had clues to malignancy and in this case these included asymmetry, a blue structureless zone and polymorphic vessels. The presence of surface scale and the presence of white circles may be clues to squamous cell carcinoma. The dermatoscopic finding of white circles as a clue to invasive squamous cell carcinoma needs to be confirmed with the study of more cases.

\section{References}

1. Kossard S, Cook D. Pigmented squamous cell carcinoma with dendritic melanocytes. Australas J Dermatol 1997;38(3):145-7.

2. Morgan MB, Lima-Maribona J, Miller RA, et al. Pigmented squamous cell carcinoma of the skin: morphologic and immunohistochemical study of five cases. J Cutan Pathol 2000;27(8):381-6.

3. Jurado I, Saez A, Luelmo J, et al. Pigmented squamous cell carcinoma of the skin. Report of two cases and review of the literature. Am J Dermatopathol 1998;20(6):578-81.

4. Matsumoto M, Sonobe H, Takeuchi T, et al. Pigmented squamous cell carcinoma of the scrotum associated with a lentigo. Br J Dermatol 1999;141(1):132-6. 
5. Kamiya M, Maehara R, Iizuka S, et al. Pigmented squamous cell carcinoma with dendritic melanocyte colonization in the external auditory canal. Pathol Int 1999;49(10):909-12.

6. Chapman MS, Quitadamo MJ, Perry AE. Pigmented squamous cell carcinoma. J Cutan Pathol 2000;27(2):93-5.

7. Shields JA, Shields CL, Eagle RC Jr, Singh AD, Demirci H, Wolf MA. Pigmented conjunctival squamous cell carcinoma simulating a conjunctival melanoma. Am J Ophthalmol 2001;132(1):104-6.

8. Terada T, Yamagami J, Fugimoto A, Tanaka K, Sugiura M. Pigmented squamous cell carcinoma of the cheek skin probably arising from solar keratosis. Pathol Int 2003;53(7):468-72.

9. Ohnishi T, Nakai K, Nagayama T, Sasaki M, Suzuki T, Watanabe S. Pigmented squamous cell carcinoma of the skin. Report of a case with epiluminescence microscopic observation. Br J Dermatol 2003;149(6):1292-3.

10. Zalaudek I, Citarella L, Soyer HP, Hofmann-Wellenhof R, Argenziano G. Dermoscopy features of pigmented squamous cell carcinoma: a case report Dermatol Surg 2004;30(4 Pt 1):539-40.

11. Satter EK. Pigmented squamous cell carcinoma. Am J Dermatopathol 2007;29(5):486-9.

12. de Giorgi V, Alfaioli B, Papi F, et al. Dermoscopy in pigmented squamous cell carcinoma. J Cutan Med Surg 2009;13(6):326-9.

13. Yoshida Y, Yamasaki A, Shiomi T, Suyama Y, Nakayama B, Yamamoto O. Ulcerative pigmented squamous cell carcinoma in a 101-year-old Japanese woman. J Dermatol 2009;36(4):241-4.

14. Cameron A, Rosendahl C, Tschandl P, Riedl E, Kittler H. Dermatoscopy of pigmented Bowen's disease. J Am Acad Dermatol 2010;62(4):597-604.

15. Kittler H. Dermatoscopy: Introduction of a new algorithmic method based on pattern analysis for diagnosis of pigmented skin lesions. Dermatopathology: Practical \& Conceptual 2007;13(1):3.

16. Kittler H, Riedl E, Rosendahl C, Cameron A. Dermatoscopy of unpigmented lesions of the skin: a new classification of vessel morphology based on pattern analysis. Dermatopathology: Practical \& Conceptual 2007;14(4):3.

17. Rosendahl C, Tschandl P, Cameron A, Kittler H. Diagnostic accuracy of dermatoscopy for melanocytic and non-melanocytic pigmented lesions. J Am Acad Dermatol 2011;64(6):1068-73.

18. Weismann K, Lorentzen HF. Dermoscopic color perspective. Arch Dermatol 2006;142(9):1250. 